\title{
Effect of Urethane Foam Cushioning on Structure-Borne Sound Transfer by a Slab with Panel Flooring
}

\author{
RuiLin MU ${ }^{(1)}$, Masahiro TOYODA ${ }^{(2)}$, Daiji TAKAHASHI ${ }^{(3)}$ \\ (1) School of Mechanical Engineering, TianJin University of Science and Technology \\ 1038, Dagu Nanlu, Hexi District, Tianjin, P. R. China; e-mail: mrl3667@tust.edu.cn \\ (2) Faculty of Environmental and Urban Engineering, Kansai University \\ 3-3-35, Yamate-cho, Suita-shi, Osaka, Japan; e-mail: toyoda@kansai-u.ac.jp \\ (3) Graduate School of Engineering, Kyoto University \\ C1-4-390, Katsura, Nishikyo-ku, Kyoto, Japan; e-mail: tkhs@archi.kyoto-u.ac.jp
}

(received May 7, 2015; accepted November 4, 2015)

\begin{abstract}
Urethane foam mattresses are commonly used as cushioning when placing panel flooring on the floor slab of a building. Urethane foam consists of elastic fibres with pores. Both elements can affect the performance of the insulation against impact sounds. However, these effects have not yet been detailed, and they may change if the material properties or constitution of the fibres and pores in the cushioning change. In this paper, we propose an analytical model for use in evaluating the performance of insulation against floor impact sound. This model was used to examine the contribution of the pores versus the elastic fibres to wave transmissions from the flooring surface to the slab. The results reveal that the constitution of the foam (either open or closed cells of pores) and the thickness and hardness of the cushion layer strongly affect the sound insulation performance of the floor.
\end{abstract}

Keywords: urethane foam; direct pasted flooring; floor impact sound; sound pressure level; sound insulation performance.

\section{Introduction}

Building quality is affected by several elements such as the design, safety, and residential amenities. Sound insulation is an important criterion for evaluating residential amenities. Many studies have attempted to improve the sound insulation performance in buildings. Typical problems related to sound insulation in buildings are sound transmission by windows and walls, floor impact sounds, and general structure-borne sounds arising from vibrations induced by equipment and machinery. The main physical manifestations of these problems are believed to be vibrating building elements and acoustic coupling with the surrounding air. A basic measure to improve sound transmission problems with a homogeneous single partition is to increase the mass and thickness. However, this approach is limited and outdated. Therefore, inhibiting sound transmission using a double-plate system with various constituents has been studied. Sound transmission through double plates enclosing an air cavity is the simplest case.
A typical example was presented by LONDON (1950), and several studies have been reported since then. Sound transmission problems for double plates with an absorptive layer have also been the subject of numerous papers; the absorptive layer is treated as a wave propagation medium with resistance dissipation. Kropp and ReBILlard (1999) examined a sandwich construction composed of double plates with an elastic solid core modelled to exhibit locally reactive elasticity (Winkler-type material). ForD et al. (1967) developed a more rigorous model for the core based on the general elastic theory. A double-plate structure with studs was designed as a lightweight construction material. These studs have an effect of shortcircuit transmissions and provide cavity boundaries. Studies on this problem have been devoted to the effects of studs with an air layer (LiN, GARRELICK, 1977; WANG et al., 2005; BRUNSKOG 2005) or an absorptive layer (Bradley, Birta, 2001a; 2001b; Brunskog, DAvidsson, 2004). Hongisto (2006) reviewed these works to compare prediction models. The structure treated here uses two plates bounded to the elastic 
core (urethane foam). In this case, the core affects wave transmission via the frame elasticity and wave propagation medium of the pores. Wave propagation theory in such a porous elastic material was established by Biot (1956) and discussed by Allard (1993). Examples using such a core in a double-plate system for sound transmission problems were presented by Bolton et al. (1996) and SGARD et al. (2000).

The aim of the present study was to clarify the fundamental mechanism of wave transmission through the core, especially the contribution of the urethane foam frame versus its pores for cushioning floor impact sounds. The frame was modelled as a Winklertype frame, and the pores were characterized as a wave propagation medium with dissipation. The model was used to compare the reductions in floor impact sounds and the relative contribution of the pores versus the elastic fibres for wave transmission from the flooring surface to the slab. The effect of the layer material on the sound insulation performance of the floor was also investigated.

\section{Analysis}

Figure 1 introduces the analytical model used to investigate the reduction in floor impact sound due to the flooring system. Here, a flooring system composed of the flooring panel and cushion is directly connected to a floor slab. This model considers the multilayer structure to have simply supported edges set in an infinite rectangular duct of size $a \times b\left(\mathrm{~m}^{2}\right)$ on an $x-y$ coordinate axis. From the source side, the flooring panel located at $z=0$ is excited by a point force, $Q_{0} e^{-i \omega t}$, at $\left(x_{0}, y_{0}\right)$. The second layer is a urethane foam cushion with a thickness of $L$. The inner surface of the duct is assumed to be rigid. Both sides of this structure and the pores of the urethane foam cushion are considered to be filled with the same fluid (air). Additionally, the time factor is in the steady state, $\exp (-i \omega t)$, where $\omega$ is the angular frequency; $k$ represents the wave number of air, and $i$ is an imaginary unit.

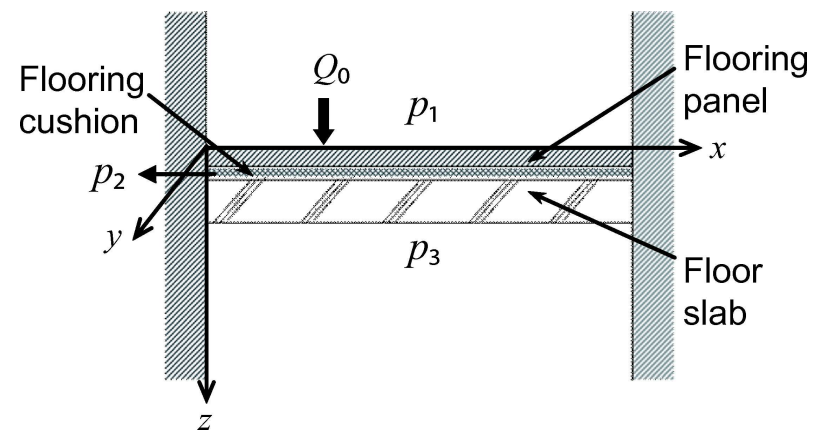

Fig. 1. Analytical model for a slab with panel flooring excited by a point force $Q_{0}$.

The sound pressure, $p$, in each region of this structure and the displacement of the flooring panel and floor slab, $w$, can be expressed using a series of expansions for each eigenfunction with unknown coefficients $A_{m n}, B_{m n}, C_{m n}, D_{m n}$, and $W_{j M N}(\mathrm{MU}, 2011)$ :

$$
\begin{aligned}
& p_{1}= \sum_{m n} A_{m n} \varphi_{m n}(x, y) e^{-i k \beta_{m n} z}, \\
& p_{2}= \sum_{m n} B_{m n} \varphi_{m n}(x, y) e^{-q_{m n} z} \\
&+\sum_{m n} C_{m n} \varphi_{m n}(x, y) e^{q_{m n} z}, \\
& p_{3}= \sum_{m n} D_{m n} \varphi_{m n}(x, y) e^{i k \beta_{m n} z}, \\
& w_{j}= \sum_{M N} W_{j M N} \psi_{M N}(x, y), \\
& \beta_{m n}=\left\{\sqrt{1-\left(\lambda_{m n} / k\right)^{2}}, \quad\left(\lambda_{m n}^{2} \leq k^{2}\right),\right. \\
& i \sqrt{\left(\lambda_{m n} / k\right)^{2}-1}, \quad\left(\lambda_{m n}^{2}>k^{2}\right), \\
& \lambda_{m n}^{2}=(m \pi / a)^{2}+(n \pi / b)^{2}, \\
& q_{m n}= \gamma \sqrt{1+\left(\lambda_{m n} / \gamma\right)^{2}} .
\end{aligned}
$$

Here, $q_{m n}$ is a variable limited by $\operatorname{Re}\left\{q_{m n}\right\} \geq 0$ and $\operatorname{Im}\left\{q_{m n}\right\} \leq 0 ; \gamma$ is the propagation constant for the urethane foam. The flooring panel and floor slab are identified by the subscript $j(=1,2)$. The eigenfunctions are $\varphi_{m n}=\cos (m \pi x / a) \cos (n \pi y / b)(m, n=$ $0,1,2, \ldots), \psi_{M N}=\sin (M \pi x / a) \sin (N \pi y / b)(M, N=$ $1,2,3 \ldots)$. The function $\varphi_{m n}$ satisfies the following orthogonal condition:

$\int_{a}^{0} \int_{b}^{0} \varphi_{m n} \varphi_{m^{\prime} n^{\prime}} \mathrm{d} x \mathrm{~d} y=\left\{\begin{array}{c}\frac{a b}{4} \varepsilon_{m} \varepsilon_{n}, m=m^{\prime}, n=n^{\prime} \\ 0, \quad \text { otherwise }\end{array}\right.$

where

$$
\varepsilon_{m}=\left\{\begin{array}{r}
2, m=0, \\
1, m \neq 0,
\end{array} \quad \varepsilon_{n}=\left\{\begin{array}{r}
2, n=0 \\
1, n \neq 0 .
\end{array}\right.\right.
$$

Notably, the eigenfunction for air in the $x-y$ plane differs from the eigenfunction for the panels. Solving these simultaneous linear equations requires a direct correlation between the two eigenfunctions $\varphi_{m n}$ and $\Psi_{M N}$. The relation is written below:

$$
\Psi_{M N}(x, y)=\sum_{m n} \alpha_{M N}^{m n}(x, y) \varphi_{m n}
$$

where

$$
\begin{aligned}
& \alpha_{M N}^{m n}=\left(\varsigma_{M m_{1}}+\varsigma_{M m_{2}}\right)\left(\varsigma_{N n_{1}}+\varsigma_{N n_{2}}\right) / \varepsilon_{m} \cdot \varepsilon_{n}, \\
& \varsigma_{M m_{1}}=\left\{\begin{array}{cc}
0, & (M+m-1=0), \\
\frac{1-(-1)^{M+m-1}}{(M+m-1) \pi}, & (M+m-1 \neq 0),
\end{array}\right.
\end{aligned}
$$




$$
\begin{gathered}
\varsigma_{M m_{2}}=\left\{\begin{array}{cc}
0, & (M-m+1=0), \\
\frac{1-(-1)^{M-m+1}}{(M-m+1) \pi}, & (M-m+1 \neq 0),
\end{array}\right. \\
\varsigma_{N n_{1}}=\left\{\begin{array}{cc}
0, & (N+n-1=0), \\
\frac{1-(-1)^{N+n-1}}{(N+n-1) \pi}, & (N+n-1 \neq 0),
\end{array}\right. \\
\varsigma_{N n_{2}}=\left\{\begin{array}{cc}
0, & (N-n+1=0), \\
\frac{1-(-1)^{N-n+1},}{(N-n+1) \pi} & (N-n+1 \neq 0) .
\end{array}\right.
\end{gathered}
$$

The effect of the flooring cushion is divided into two individual parts in the calculation: the effect of the elastic fibres and the effect of the pores. $w_{1}$ is the displacement of the flooring panel, and $w_{2}$ is the displacement of the floor slab. $\sigma$ is the stress on the flooring cushion. The force relation of the elastic fibres can be expressed as follows:

$$
\begin{gathered}
\sigma=E \xi+C \frac{\partial \xi}{\partial t} \\
\xi=\frac{w_{1}-w_{2}}{L} .
\end{gathered}
$$

Here, Young's modulus, $E\left(\mathrm{~N} / \mathrm{m}^{2}\right)$, is directly related to the hardness. $C$ and $\xi$ are the damping coefficient and strain, respectively. The stress can be written from the two equations above as follows:

$$
\sigma=\frac{E^{*}\left(w_{1}-w_{2}\right)}{L}
$$

where

$$
E^{*}=E(1-i \eta)
$$

$E^{*}$ represents the complex Young modulus, and $\eta$ is the loss factor.

After accounting for the effects of the elastic fibres and pores on the cushion, the equations of motion for the panel displacements, $w_{1}(x, y)$ and $w_{2}(x, y)$, can be expressed as follows:

$$
\begin{aligned}
D_{1} \nabla^{4} w_{1}-\rho_{1} h_{1} \omega^{2} w_{1}= & \left.p_{1}\right|_{z=0}-\left.p_{2}\right|_{z=0} \\
& -\frac{E\left(w_{1}-w_{2}\right)}{L} \\
& +q_{0} \delta\left(x-x_{0}\right) \delta\left(y-y_{0}\right), \\
D_{2} \nabla^{4} w_{2}-\rho_{2} h_{2} \omega^{2} w_{2}= & \left.p_{2}\right|_{z=L}-\left.p_{3}\right|_{z=L} \\
& +\frac{E\left(w_{1}-w_{2}\right)}{L},
\end{aligned}
$$

where $\nabla^{4}=\left(\partial^{2} / \partial x^{2}+\partial^{2} / \partial y^{2}\right)^{2}, D$ and $\rho$ are the flexural rigidity $(\mathrm{Nm})$ and density $\left(\mathrm{kg} / \mathrm{m}^{3}\right)$, respectively; $h_{j}(j=1,2)$ is the panel thickness $(\mathrm{m}) ; \delta$ represents the delta function. The second term on the right-hand side of Eq. (20) is the effect of the pores. The third term is the effect of the elastic fibres.
The boundary conditions at the interface of each layer can be expressed by the particle velocities, $v_{1}$ and $v_{2}$, and the panel displacements, $w_{1}$ and $w_{2}$, as follows:

$$
\begin{aligned}
\left.v_{1}\right|_{z=0} & =\left.v_{2}\right|_{z=0}, \\
\left.v_{1}\right|_{z=0} & =-i \omega w_{1}, \\
\left.v_{2}\right|_{z=L} & =\left.v_{3}\right|_{z=L}, \\
\left.v_{3}\right|_{z=L} & =-i \omega w_{2} .
\end{aligned}
$$

Substituting the expanded sound pressure, particle velocity, and displacement for the panel into Eqs. (20), (21) and coupling with the boundary condition yields simultaneous equations for unknown coefficients $A_{m n}$, $B_{m n}, C_{m n}, D_{m n}$, and $W_{j m n}(j=1,2)$, which can be solved numerically. The acoustic power, $P$, radiated from this structure into the transmitted side can be calculated as follows:

$$
\begin{aligned}
P & =\int_{S} \frac{1}{2} \operatorname{Re}\left\{p_{3}(x, y, L) \cdot v_{3}^{*}(x, y, L)\right\} \mathrm{d} S \\
& =\frac{a b}{8 \rho_{0} c_{0}} \sum_{m n} \varepsilon_{m} \varepsilon_{n}\left|D_{m n}\right|^{2} \beta_{m n} .
\end{aligned}
$$

Here, * represents the complex conjugate and $S$ is taken over the area $a \times b . \rho_{0}$ and $C_{0}$ are the air density and sound speed in air, respectively. The sound insulation performance of the floor structure is evaluated based on the difference in power level with and without the flooring system.

\section{Discussion of the layer model}

To investigate the reduction in floor impact sound due to the flooring system, experiments were conducted using a simplified method to measure the floor impact sound with a light impact source. Figure 2 shows the measurement system. The flooring system was installed over a $930 \times 905 \mathrm{~mm}^{2}$ area. A 9-mmthick flooring panel was connected to a $150-\mathrm{mm}$ slab in a reverberation room via a urethane foam cushion. The urethane foam was $4 \mathrm{~mm}$ thick. A standard tapping machine on the specimen was used as the impact
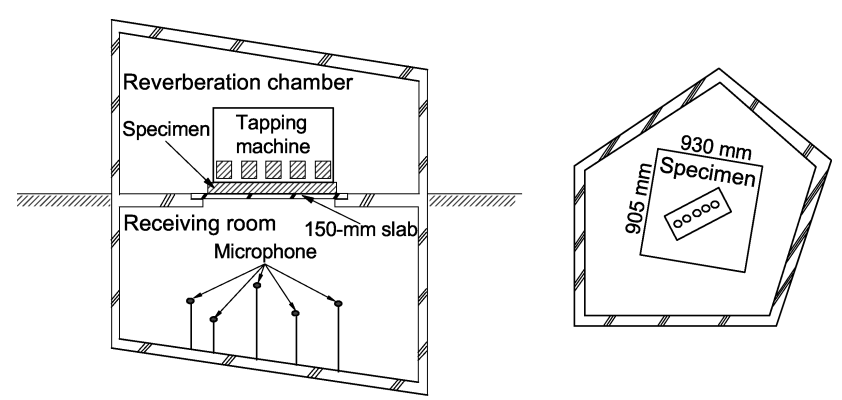

Fig. 2. Configuration of the measurement system (coupled reverberation rooms and test specimen of flooring with a tapping machine). 
source. The sound pressure level in the receiving room was measured at five positions across the $1 / 1$ octave band and averaged; this value is denoted as $L_{F}(\mathrm{~dB})$. The SPL without flooring was measured as the reference level $L_{R}(\mathrm{~dB})$. The reduction in impact sound, $\Delta L(\mathrm{~dB})$, due to the flooring was defined as the difference with and without the flooring system. The panel size does not significantly affect $\Delta L$ for limp panels with a soft urethane foam cushion. The corresponding calculations were performed under these measurement conditions using a $3 \times 4 \mathrm{~m}^{2}$ double-plate system $(a=$ $3 \mathrm{~m}, b=4 \mathrm{~m})$. The excitation point in the calculation was taken at an off-centre point $(3 a / 8,3 b / 8)$ to enable many modes to be involved. Any size beyond this as well as changes in the excitation point were confirmed to have less effect on $\Delta L$ when averaged in the $1 / 1$ octave band. The properties of the flooring layer materials are presented in Table 1 . The hardness of the urethane foam was measured following JIS K6400 (2004).

Table 1. Material properties of the flooring layer.

\begin{tabular}{|c|c|c|c|c|}
\hline Layer & $\begin{array}{c}\text { Young's } \\
\text { modulus } \\
{\left[\mathrm{N} / \mathrm{m}^{2}\right]}\end{array}$ & $\begin{array}{c}\text { Density } \\
{\left[\mathrm{kg} / \mathrm{m}^{3}\right]}\end{array}$ & $\begin{array}{c}\text { Poisson } \\
\text { ratio }\end{array}$ & $\begin{array}{c}\text { Loss } \\
\text { factor }\end{array}$ \\
\hline plywood panel & $5.4 \times 10^{9}$ & 600 & 0.3 & 0.01 \\
\hline urethane foam & $\begin{array}{c}60 \mathrm{~N} \\
(\text { hardness })\end{array}$ & - & - & 0.1 \\
\hline concrete & $2.7 \times 10^{10}$ & 2300 & 0.2 & 0.005 \\
\hline
\end{tabular}

The experimental results were compared using analytical calculations to examine the role the elastic fibres and pores have as wave transmission elements. In the calculation, five simple cases were considered to determine the effect of the pores.

The first case only considered the elastic fibres in the calculation. In other words, the effect of the pores was neglected, which is an extreme case. Figure 3 shows the calculated result for the sound power level

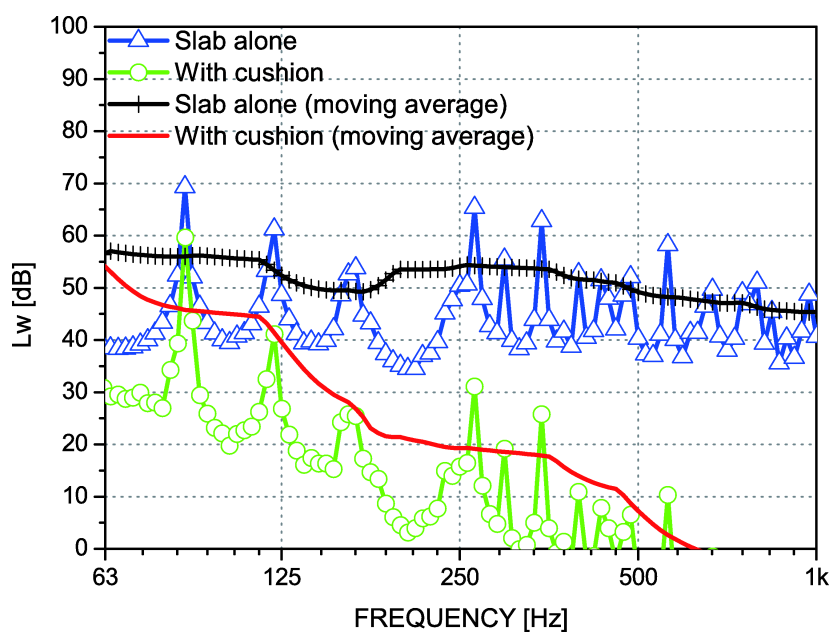

Fig. 3. Comparison of the power levels with and without the flooring system (elastic fibres only).
$\left(L_{w}\right)$ on the transmitted side caused by a $1 \mathrm{~N}$ force. The blue (- $\left.\triangle_{-}\right)$and black (-+-) lines represent the results for the slab alone, and the green (- $\bigcirc-)$ and red (solid) lines show the results for the case with the flooring system. The blue and green lines are the raw data, and the black and red lines are the moving average for the $1 / 1$-octave band interval.

The second case only considers wave transmission due to pores. In other words, the effect of the elastic fibres was neglected, and the effects of the pores were considered to be sound waves propagating in air, which is another extreme case. The purpose of this case was to compare the differences between the different models and investigate the effects of pores. Figure 4 shows the calculated results with and without the flooring system.

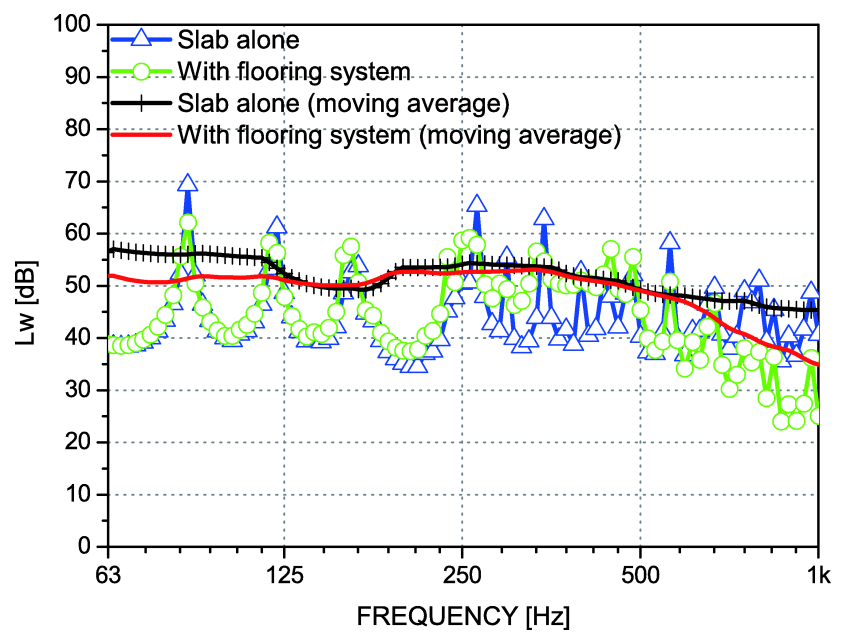

Fig. 4. Comparison of the power levels with and without the flooring system (pores only).

In the third case, both elements were considered equally. The calculated results are shown in Fig. 5. This model investigated the effect of the pores on the calculations for the porous materials.

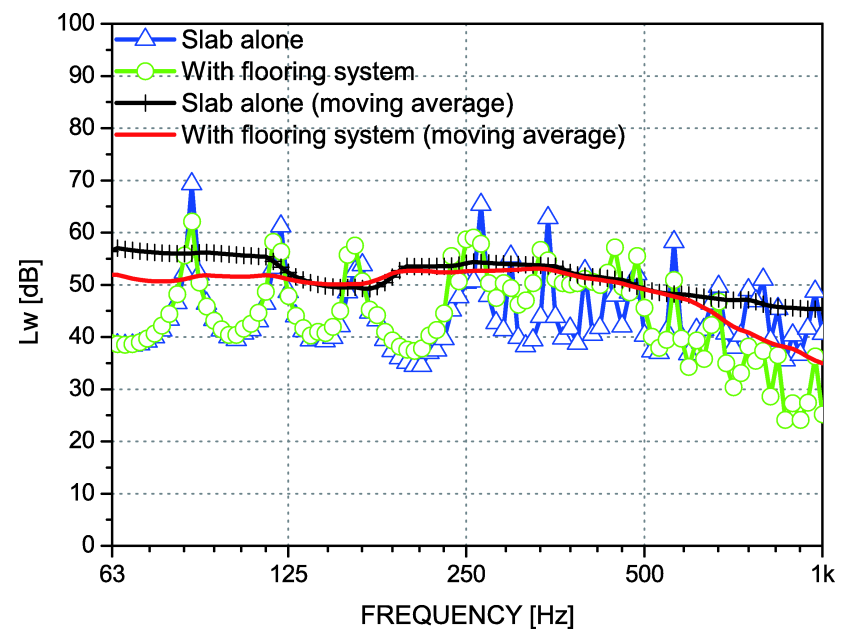

Fig. 5. Comparison of the power levels with and without a flooring system (elastic fibres and pores are equal). 
In the fourth case, the wave propagation model of pores was replaced with an equivalent spring model. The pores were treated as a spring without energy loss. The equivalent spring model is shown in Fig. 6. The variation in pore volumes is considered to be the spring variation under this assumption.
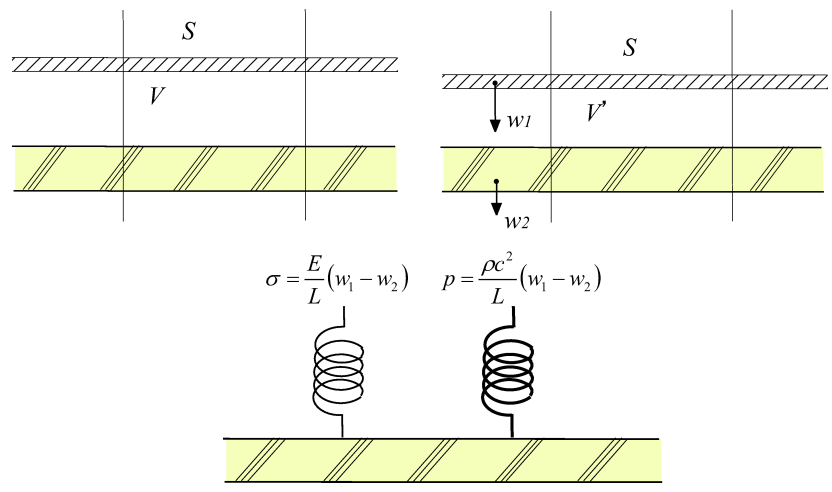

Fig. 6. Equivalent spring model.

Under these conditions, the equivalent spring equation can be expressed as follows:

$p=-K \frac{V^{\prime}-V}{V}=\frac{K}{L}\left(w_{1}-w_{2}\right)=\frac{\rho c^{2}}{L}\left(w_{1}-w_{2}\right)$.

Figure 7 compares the calculated results for the fourth case.

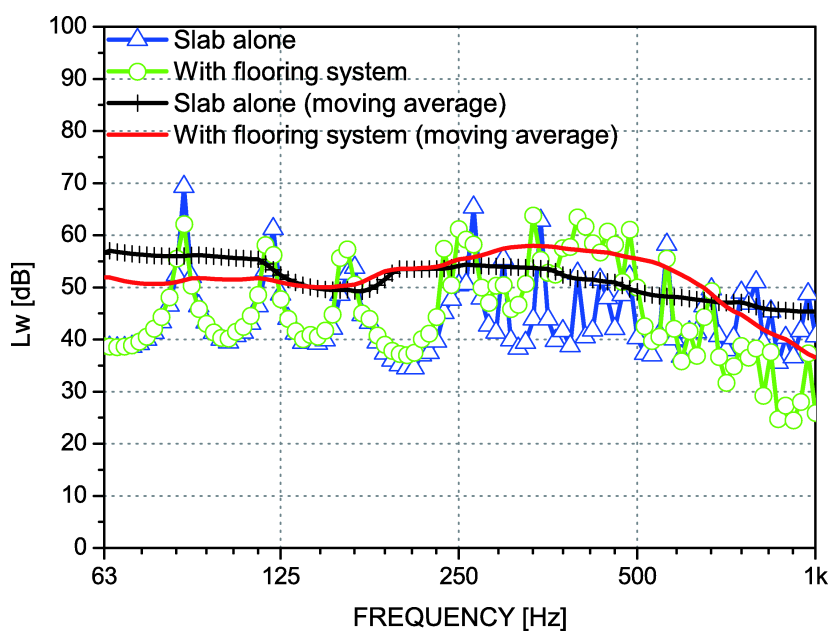

Fig. 7. Comparison of the power level with and without the flooring system (equivalent spring model instead of wave propagation model of pores).

Figure 8 compares the $\Delta L$ for the calculated and experimental results. The green line $(-\bullet-)$ is the calculated result of the first case, the black dotted line $(.$.$) is the calculated result of the second case, the$ black solid line is the experimental result. While the calculated result of the third case is the red line $(-\boldsymbol{-})$, the blue line (- $\mathbf{\Delta}-)$ presents the calculated result of the fourth case.

The calculated results were similar for the second through fourth cases but very different in the first

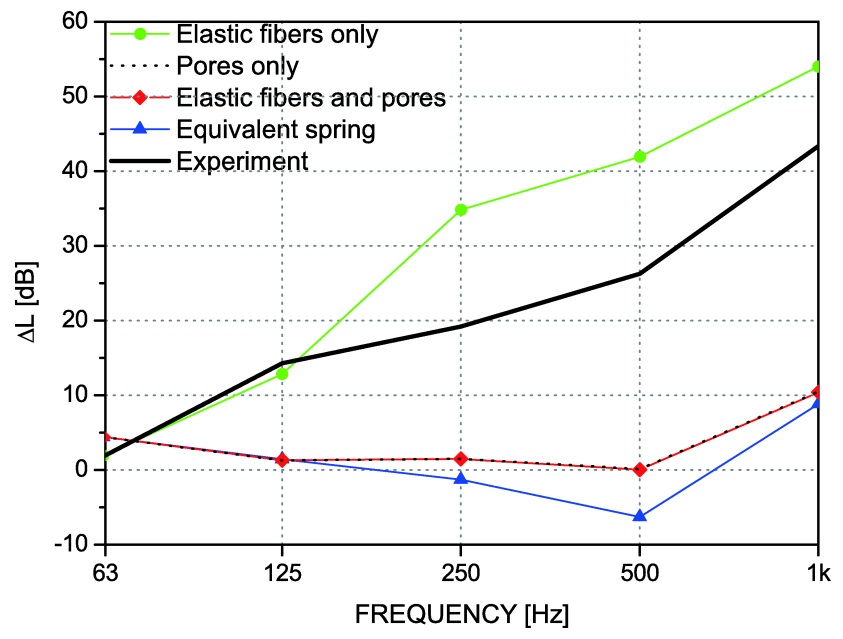

Fig. 8. Comparison of the theoretical and experimental values of $\Delta L$.

case (elastic fibres alone). The deviation between the green line (-•-) and black solid lines (the first case) was approximately $10 \mathrm{~dB}$ for the frequencies from 200 to $1000 \mathrm{~Hz}$. However, the deviation for the cases two through four was over $13 \mathrm{~dB}$. The max deviation was approximately $25 \mathrm{~dB}$ for the second and third cases, whereas the max deviation in the fourth case reached approximately $30 \mathrm{~dB}$. Because the first case did not include pore effects, the calculated results are closer to the experimental results. These results indicate that pores strongly affect the calculations for porous materials. In other words, the pores play a more important role than the elastic fibres when calculating the effect of a porous material.

The above results showed that the results calculated using the proposed analytical model without any change to the contribution ratio of the wave transmission of pores to the elastic fibres do not agree well with the experimental results. However, changing the contribution ratio from the pores to $2 \%$ caused the calculated results to align with the experimental results. The calculated results and $\Delta L$ between the theoretical and experimental results for this fifth case are shown in Figs. 9 and 10, respectively. A contribution ratio of $2 \%$ means that the second term on the right-hand side of Eq. (20) and the first term on the right-hand side of Eq. (21) are multiplied by 0.02 . The disparity between the theoretical and experimental values, $\Delta L$, increased as the contribution ratio of pores increased when comparing all cases for $\Delta L$. For the equivalent spring model, in which the pores were modelled as isolated cells, the disparity between the theoretical and experimental results was the highest; however, the wave propagation model showed similar results. Thus, treating the pores as a wave propagation medium is almost the same as treating them equivalently to springs when the thickness of the porous layer is smaller than the wavelength. When pores are considered as the only contributor, the theoretical 


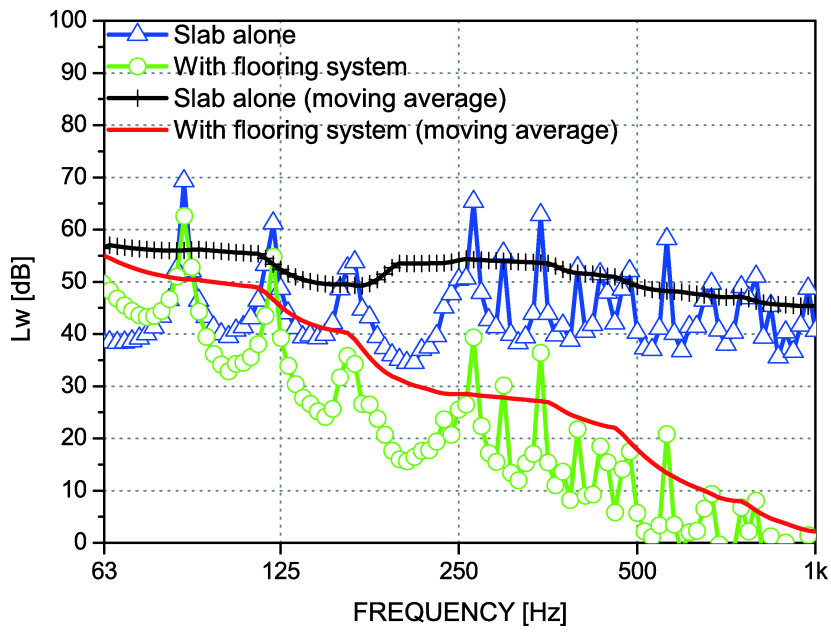

Fig. 9. Comparison of the power level with and without the flooring system (pore contribution $=2 \%$ ).

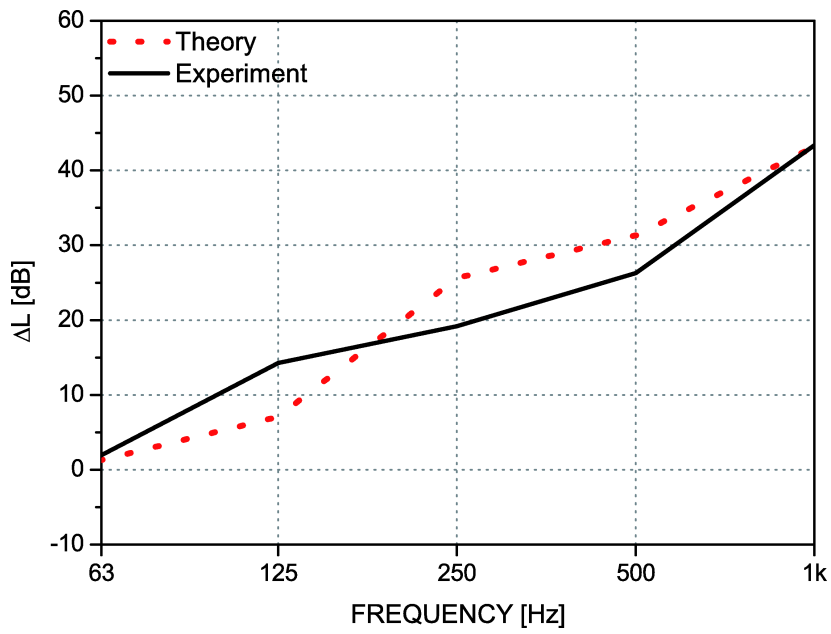

Fig. 10. Comparison of the theoretical and experimental values of $\Delta L$ (pore contribution $=2 \%$ ).

and experimental values do not agree. This difference may imply that some additional effect reduces the wave transmission in such thin wave transmission medium layers. Although a $2 \%$ contribution pore ratio was used for the calculation, this assumption requires clarification to establish an effective analytical model, which will be examined in future work. In the following section, the effect of the cushion is evaluated using a contribution ratio of $2 \%$ from pores.

\section{Effect of the layer material}

The effects of varying the thickness and hardness of the cushion were investigated. Figures 11 and 12 show the calculated results. The floor impact sound was reduced significantly by increasing the cushion thickness. In contrast, a less significant change was observed when the cushion hardness was varied. This result indicates that the sound insulation performance can be improved by softening or thickening the cushion.

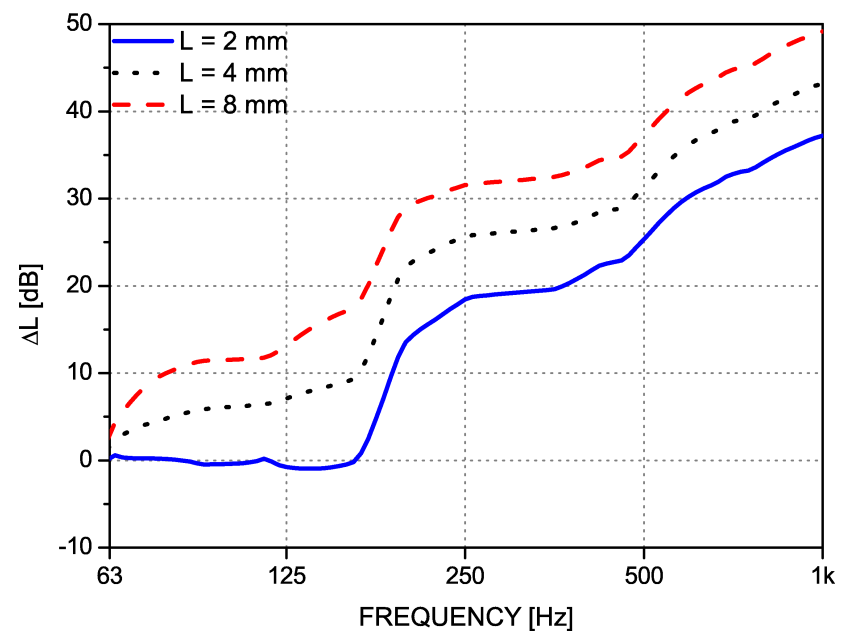

Fig. 11. Effects of varying cushion thickness on $\Delta L$.

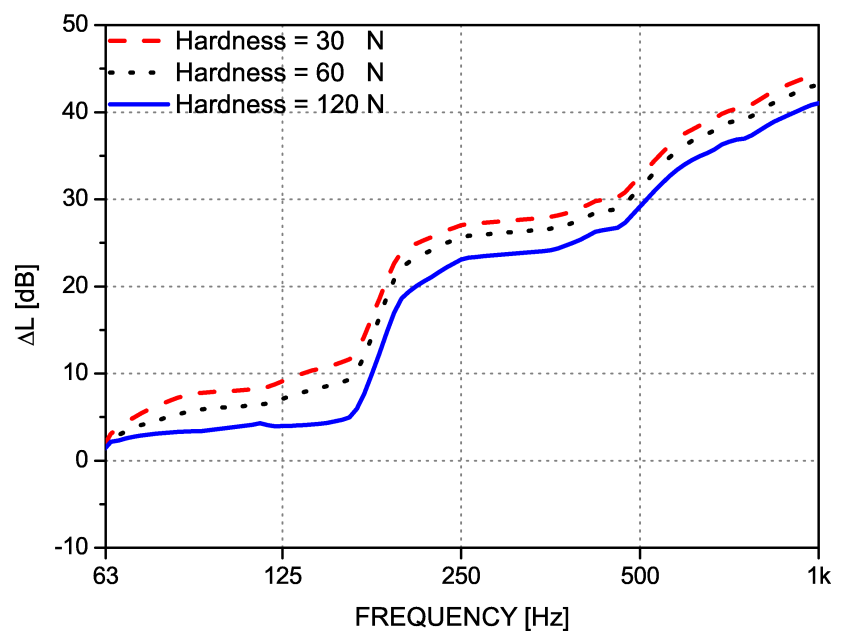

Fig. 12. Effects of varying cushion hardness on $\Delta L$.

The effect of varying the thickness and hardness on the feeling of walking over the floor was also considered; the reduced floor impact sound was examined for a constant hardness-to-thickness ratio. Figure 13

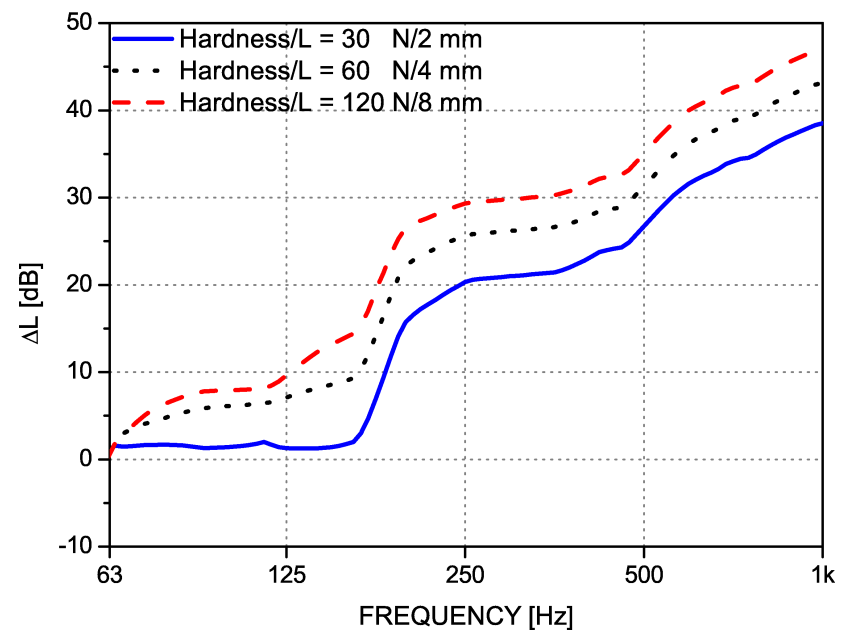

Fig. 13. Effect of changing both the hardness and thickness of the cushion at a constant ratio on $\Delta L$. 
shows the results, which were similar to the thickness results. The $\Delta L$ shifted to the safe side when both the thickness and hardness of the cushion increased at a constant ratio.

Figure 14 shows the effect of flow resistance. Increasing the flow resistance somewhat diminished the sound insulation performance at low frequencies despite the $\Delta L$ becoming larger across the range from 80 to $1000 \mathrm{~Hz}$. There was no significant change in $\Delta L$ from 80 to $1000 \mathrm{~Hz}$ when the flow resistance exceeded a certain value. In this case, the value was approximately $5000 \mathrm{Ns} / \mathrm{m}^{4}$. In addition, the effect of the loss factor was investigated, as shown in Fig. 15. There was no significant change upon increasing the loss factor; all the lines nearly overlapped. Therefore, the loss factor can be neglected.

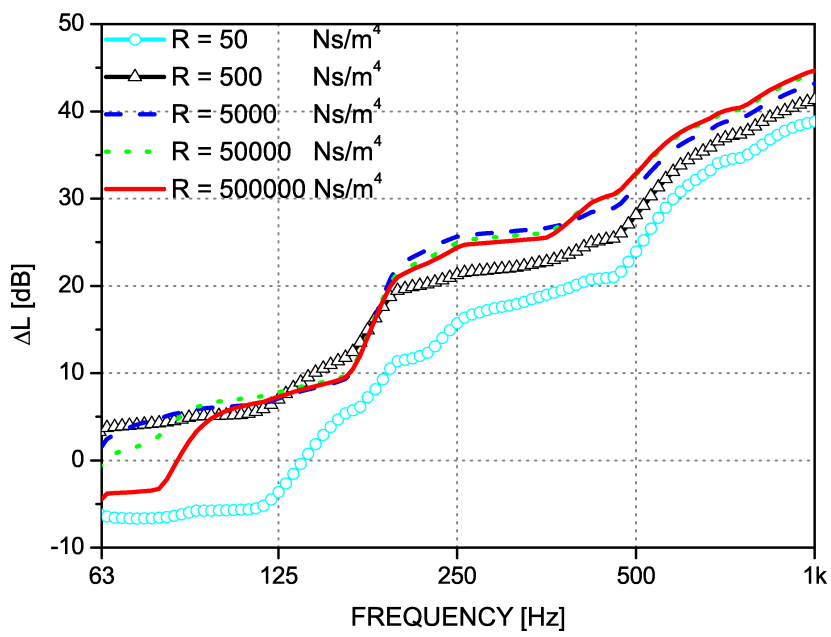

Fig. 14. Effects of varying the flow resistance of the cushion on $\Delta L$.

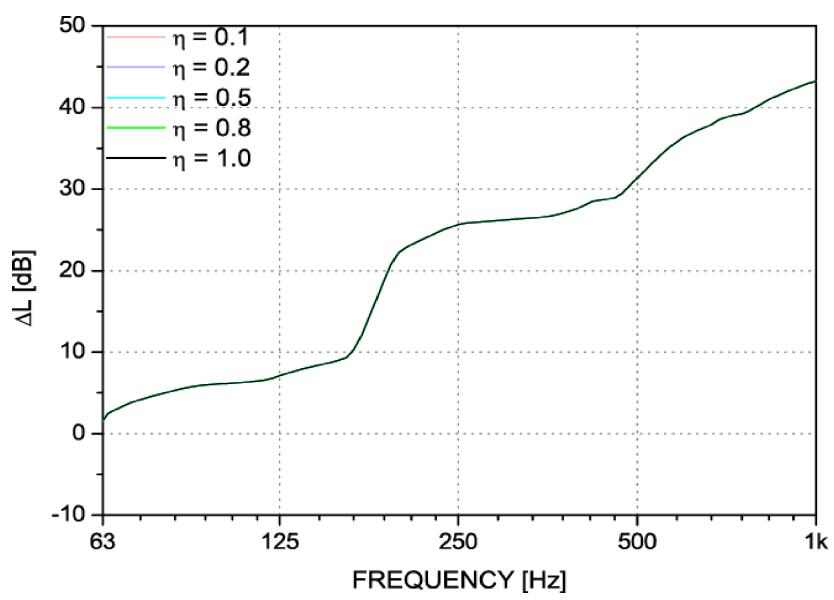

Fig. 15. Effects of varying the loss factor on $\Delta L$.

\section{Conclusions}

This study examined four hypothetical cases to investigate the effect of porous materials on the sound insulation performance of a slab with panel flooring for lightweight impact sound by comparing modelling results with the measured data. This investigation showed that the pores of the wave propagation medium strongly affect the calculations for porous materials. In other words, the pores play a more important role than the elastic fibres when calculating the effects of porous materials on sound insulation. The concept of a contribution ratio for the pores was introduced to evaluate the sound insulation performance using the proposed analytical model for lightweight floor impact sounds. The contribution ratio of pores to elastic fibres in wave transmissions from the flooring surface to the slab was investigated to compare the difference in floor impact sound reduction between the experimental and calculated results. The results calculated using a $2 \%$ contribution from the pores agreed well with the experimental results.

Furthermore, the effects of the material properties (thickness, hardness, flow resistance, and loss factor) of the porous material on the insulation performance of the floor were studied assuming a pore contribution ratio of $2 \%$ relative to the elastic fibres. A parametric survey of the changes in the properties of the urethane foam cushion indicated that the sound insulation performance can be improved by softening or thickening the cushion. Changing both the hardness and thickness at a constant ratio provides an effective improvement.

\section{Acknowledgments}

This study is supported by Tianjin University of Science and Technology and Tianjin Key Laboratory of Integrated Design and On-line Monitoring for Light Industry \& Food Machinery and Equipment. The authors also would like to thank Kyoto University for their support.

\section{References}

1. Allard J.F. (1993), Propagation of sound in porous media, p. 118-144, Elsevier Applied Science, London and New York.

2. Biot M.A. (1956), Theory of propagation of elastic waves in a fluid-saturated porous solid. I. Low Frequency Range, Journal of the Acoustical Society of America, 28, 2, 168-178.

3. Вiот M.A. (1956), Theory of propagation of elastic waves in a fluid-saturated porous solid. II. Higher Frequency Range, Journal of the Acoustical Society of America, 28, 2, 179-191.

4. Bolton J.S., Shiau N.-M., Kag Y.J. (1996), Sound Transmission through multi-panel structures lined with elastic porous materials, Journal of Sound and Vibration, 191, 3, 317-347.

5. Bradley J.S., Birta J.A. (2001), On the sound insulation of wood stud exterior walls, Journal of the Acoustical Society of America, 110, 6, 3086-3096. 
6. Bradley J.S., Birta J.A. (2001), A simple model of the sound insulation of gypsum board on resilient supports, Noise Control Engineering Journal, 49, 5, 217223 .

7. Brunskog J. (2005), The influence of finite cavities on the sound insulation of double-plate structures, Journal of the Acoustical Society of America, 117, 6, 37273739 .

8. Brunskog J., Davidsson P. (2004), Sound transmission of structure. A finite element approach with simplified room description, Acta Acustica united with Acustica, 90, 5, 847-857.

9. Ford R.D., Lord P., Williams P.C. (1967), The Influence of absorbent linings on the transmission loss of double-leaf partitions, Journal of Sound and Vibration, $\mathbf{5}, 1,22-28$.

10. Hongisto V. (2006), Sound insulation of double panels - Comparison of existing prediction models, Acta Acustica united with Acustica, 92, 1, 61-78.

11. JIS K6400-2 (2004), Flexible cellular polymeric materials - Determination of the physical properties Part 2: Hardness (indentation technique) and stressstrain characteristics in compression.
12. Kropp W., Rebillard E. (1999), On the air-borne sound insulation of double wall constructions, Acta Acustica united with Acustica, 85, 5, 707-720.

13. Lin G.-F., Garrelick J.M. (1977), Sound transmission through periodically framed parallel plates, Journal of the Acoustical Society of America, 61, 4, 1014-1018.

14. LONDON A. (1950), Transmission of reverberant sound through double walls, Journal of the Acoustical Society of America, 22, 2, 270-279.

15. Mu R.L., Toyoda M., Takahashi D. (2012), Improvement of sound insulation performance of doublepanel structures by using damping materials, Noise Control Engineering Journal, 60, 4, 473-480.

16. Sgard F.C., Atalla N., Nicolas J. (2000), $A$ numerical model for the Low Frequency diffuse field sound transmission loss of double-wall sound barriers with elastic porous linings, Journal of the Acoustical Society of America, 108, 6, 2865-2872.

17. Wang J., Lu T.J., Woodhouse J., Langley R.S., Evans J. (2005), Sound transmission through lightweight double-leaf partitions: Theoretical modelling, Journal of Sound and Vibration, 286, 4-5, 817-847. 